\title{
La actividad emprendedora en la provincia de El Oro, una revisión desde la universidad
}

\section{Entrepreneurship in the province of El Oro, a review from the university}

Jhon Campuzano Vásquez, Mgs

Patricia Uriguen Aguirre, MBA

Johanna Pizarro Romero, Mgs

Eileen Cedeño Flores, Mgs

Grupo de Investigación GIDET

Universidad Técnica de Machala, Ecuador

Autor para correspondencia: jcampuzano@utmachala.edu.ec,puriguen@utmachala.edu.ec, jpizarro@utmachala.edu.ec, eileencede14@hotmail.com

Fecha de recepción: 14 de Junio de 2017 - Fecha de aceptación: 15 de Noviembre de 2017

Resumen: Éste artículo hace un seguimiento al emprendimiento desde el accionar de las universidades públicas y privadas de la provincia de El Oro, considera varias posiciones teóricas sobre la acción de la academia frente al emprendimiento, clasifica las principales acciones que ejecuta un centro de educación superior para promover la actividad emprendedora o situaciones que puedan ser identificadas en este sentido, y toma como referente internacional el informe GEM para probar la importancia del emprendimiento en el Ecuador al no existir información desagregada a nivel de provincia. Se recogen datos estadísticos de la Universidad Técnica de Machala y de la Universidad Metropolitana sede Machala haciendo un análisis comparativo y longitudinal que sintetiza la acción de estas dos universidades, además se recoge el hacer de instituciones públicas nacionales y locales relacionadas al fomento del emprendimiento en la provincia en los últimos cinco años, determinando que existen resultados negativos en la acción universitaria en temática emprendedora los cuales pueden ser asumidos en las investigadas IES como algo común por su débil estructura de apoyo tanto en lo técnico como en lo económico.

Palabras clave: emprendimiento; universidad; análisis; informe

\begin{abstract}
This article follows the entrepreneurship from the actions of the public and private universities of the province of El Oro, considers several theoretical positions on the action of the academy against the entrepreneurship, classifies the main actions carried out by a higher education center to promote The entrepreneurial activity or situations that can be identified in this sense, and takes as an international reference the GEM report to prove the importance of entrepreneurship in Ecuador as there is no disaggregated information at the province level. Statistical data are collected from the Technical University of Machala and the Metropolitan University Machala, making a comparative and longitudinal analysis that synthesizes the action of these two universities, in addition to collecting the national and local public institutions related to the promotion of entrepreneurship in the Province in the last five years, determining that there are negative results in university action in entrepreneurship, which can be assumed in the researched IES as something common because of its weak structure of support in both the technical and the economic.
\end{abstract}

Key words: entrepreneurship; university; analysis; report 


\section{Introducción}

Para nadie es desconocido los cambios que viene experimentando la universidad en el mundo desde el siglo diecinueve. El dinamismo de las tecnologías de la información y comunicación, la búsqueda de nuevas formas de desarrollo social, la urgencia de la rendición de cuentas a la sociedad y la vinculación en redes internacionales, son algunas de los elementos conducentes al cambio. Por lo que, ya no es posible pensar en una universidad encerrada en su territorio, más bien se observan instituciones dinámicas y con mayor presencia en las actividades productivas, sociales, culturales y deportivas en cada uno de sus lugares de acción educativa.

Un claro ejemplo es mencionado por Huanca (2004) que concibe la relación entre universidad y empresa de una manera interactiva, a la cual la denomina, estrategia de desarrollo. Castro y Vega (2009) posteriormente manifiestan que la preparación de profesionales; para ellos, ya no es el objetivo único de la universidad, más bien se vive la importancia de generar conocimiento a través de la vinculación entre enseñanza e investigación.

Con lo que los objetivos de los centros universitarios caminan a profesionalizar al estudiante mediante procesos fuertes de vinculación a la investigación empresarial, que deben coordinarse con mallas curriculares fortalecidas con prácticas investigativas y la enseñanza de conocimientos que promueven la aptitud emprendedora entre los universitarios. El gran desafío, sigue vigente y se enmarca, en el cómo la academia puede crear y difundir conocimiento y hacer del saber científico-tecnológico un bien comercializable; sin que esto se convierta en un estigma interno para los que promueven el cambio acelerado en las instituciones de educación superior, frente a los que mantienen viejas prácticas docentes e investigativas.

Incluso la creación de proyectos emblemáticos en educación superior en Ecuador, como es la puesta en marcha de la universidad Yachay Tech, confirma lo mencionado sobre la comercialización de la producción científica, sin que esta se aleje de las necesidades comunitarias o de territorios más grandes de conocimiento aplicado, que se explicita en su estatuto puntualmente en el artículo 10 literales a, c y d (Yachay Tech, 2014).

El alcanzar objetivos de promoción del cambio vía el conocimiento aplicado al emprendimiento, hace que surjan varias preguntas que pueden ser analizadas considerando la parte presupuestaria: ¿Hay falta de recursos en las universidades y en los estudiantes, y eso impide crear, difundir y comercializar conocimiento? ¿Qué está haciendo la universidad para promover la investigación vinculada a las necesidades de las organizaciones y de qué manera la aptitud emprendedora universitaria, puede ser un factor motivador para lograr objetivos de excelencia académica que demuestren calidad en los profesionales graduados?

Definitivamente estamos ante una "nueva universidad" con mayores reivindicaciones y desafíos en el campo educativo, el que debe reestructurarse internamente para responder a esos cambios (Chaparro, 2010). En este recorrido de cambio Vega, Manjarrés y Fernández de Lucio (2011) plantean la importancia de identificar la relación adecuada entre universidad y empresa, alejándose de generalidades para encontrar relaciones específicas mutuamente exitosas a las que podríamos denominar alianzas; tomando en cuenta que cada institución tiene diferentes misiones y visiones estratégicas. 
Una de estas relaciones específicas se puede ver con Poblete y Amorós (2013) que considerando a Bonaccorsi y Piccaluga (1994) al hablar de la importancia de la vinculación práctica de las entidades educativas con el contexto socioeconómico, señalan que hay una generación de múltiples beneficios para ambas partes, las empresas tienen un acceso inmediato al conocimiento científico-tecnológico y las universidades obtienen recursos de la empresa privada para el financiamiento de sus actividades de investigación, además de llevar a cabo nuevas investigaciones orientadas a la aplicación en áreas fuera de la academia.

\section{Educación universitaria y emprendimiento}

Contribuir al progreso de los pueblos por parte de la universidad, es actualmente la misión a alcanzar, y dentro de ella, la formación de profesionales con calidad investigativa y propositiva es un factor clave (Sanabria, Morales y Ortiz, 2015).

En esta misión es importante coordinar procesos internos de apoyo a iniciativas que contribuyan a la formación profesional de excelencia, una de ellas es sin duda la capacidad de emprender de los universitarios. La que para Juliá (2014) debe ser fortalecida de manera colaborativa, mediante conocimientos que fomenten y acrecienten el espíritu de la creación de nuevas empresas; dejando de lado metodologías de enseñanzas obsoletas, como la enseñanza informativa (saber) y pasar a promover la enseñanza de inteligencia ejecutiva "capacidad de hacer y actuar".

Sanabria, Morales y Ortiz (2015) concuerdan con Juliá (2014) en la necesidad de actuar colaborativamente entre instituciones para que los estudiantes puedan fortalecer sus conocimientos en emprendimiento. De nada servirá entonces, transmitir conocimientos sin que puedan ser aplicados a problemas empresariales o de la comunidad, o que respodan a una necesidad concreta dentro de un territorio.

Cardona, Morales, Cárdenas y Ramírez (2015) tomando a varios autores, mencionan que la formación en emprendimiento en los estudiantes provoca efectos positivos para aumentar su disposición para la creación de empresas y a su vez robustecer la pretensión y deseo de emprender. La sola creación de empresas no es el objetivo, sino las habilidades que se adquieren en la detección de soluciones a problemas, como por la capacidad prospectiva que se obtiene. Así mismo Cardona et al. (2015) mencionando a Fairlie y Holleran (2012) ratifican que una formación en emprendimiento eleva las posibilidades de emprender, y de éxito profesional a mediano plazo.

En esa formación emprendedora, Piguave (2014) considera que los docentes universitarios deben planificar adecuadamente sus clases para generar dinamismo que permita a todos los estudiantes el empoderamiento de conocimientos teóricos y prácticos para el perfeccionamiento de sus habilidades como nuevos emprendedores, haciendolos así innovadores y creativos. En esta línea (Thursby, 2005) manifiesta que la formación de emprendedores debe darse tanto en carreras de grado, maestría y doctorado, uniendo la realidad y los negocios con la educación. 
Concuerda con ello Pertuz, Rojas, Navarro y Quintero (2016) al vincular los procesos de enseñanza-aprendizaje con experiencias previas y con situaciones que permitan al estudiante la construcción del conocimiento mediante la interacción con el contexto práctico. Krauss (2011, p. 31) refiéndose a la educación para el emprendimiento, y lo que se debe enseñar indica:

Que el objetivo se basa en enseñar a los estudiantes diferentes aspectos sobre la creación de empresas, sobre las actitudes y los valores propios de empezar una empresa, gerenciarla y hacerla crecer. La enseñanza es desde una perspectiva teórica. Los forman para que en un futuro deseen crear su propia empresa.

Por lo que deben considerarse, el conocimiento, las habilidades y los marcos para categorizar y evaluar la información que poseen los estudiantes universitarios, a ello, hay que adicionar, las habilidades para identificar la información relevante que necesitan para proponer una actividad emprendedora, junto al marco regulatorio vigente, un tema poco topado que puede incidir en el éxito o fracaso de la propuesta. Todo lo anterior, se articula en el marco colaborativo que manifiestan Sanabria et al. (2015) y que tiene como actor principal al gobierno nacional y en algunos casos a gobiernos seccionales, con incidencia directa mediante programas de apoyo que se vinculan a las instancias educativas y empresariales, lo que se puede describir como el ecosistema base para el emprendimiento.

\section{Indicadores de la actividad emprendedora}

Medir la actividad emprendedora en un país, región, provincia o ciudad es uno de los objetivos básicos para entender al sistema de emprendimiento. La principal fuente con la que se cuenta a nivel macro es el informe GEM$^{1}$ (2015) este considera variables como: programas gubernamentales, políticas gubernamentales de regulación, apoyo financiero y normas sociales y culturales que inciden para que exista un entorno favorable para el emprendimiento. Ecuador es parte del monitoreo anual y tiene una puntuación de 6 sobre 9 , ubicándose por encima de la media total (4,5 puntos), muy buena puntuación en términos absolutos.

Adicional a los informes GEM, en los últimos años la Secretaría de Educación Superior, Ciencia, Tecnología e Innovación (SENESCYT) impulsa concursos en universidades ecuatorianas que fomentan e incentivan el emprendimiento entre los estudiantes, con lo cual se comienzan a tener datos estadísticos de la produccion emprendedora nacional desde el sistema de educación superior. También se pueden mencionar organizaciones sin fines de lucro que fomentan actividades emprendedoras, como Espiral, Startup Weekend, Girls in Tech, entre otras; las cuales se encuentran ubicadas en las principales ciudades del país como son: Quito, Guayaquil y Cuenca), que comienzan a aportar datos importantes sobre la actividad emprendedora en el país.

Para Feijoo (2015), las universidades ecuatorianas sean éstas públicas o privadas, deben tener una variada estructura que apoye el emprendimiento dentro de la formación de los estudiantes universitarios, siendo éstas: actividades de capacitación en innovación, vinculación social con la comunidad, desarrollo de actividades académicas fomentadoras de ideas de emprendimiento, emprendimientos simulados dentro de aula, ferias de innovación,

\footnotetext{
${ }^{1}$ Global Entrepreneurship Monitor
} 
departamentos dentro de las universidades que se encargan del control y planificación para el incentivo de proyectos nuevos de emprendedurismo. Lo señalado por Feijoo se puede observar en las universidades públicas y privadas ecuatorianas que comienzan a mostrar y apoyar emprendimientos, aunque no se presentan estadísticas exitosas de creación, expansión y sostenibilidad en el tiempo.

En Ecuador existen tres universidades que vienen siendo los referentes más potentes para el fomento del emprendimiento, se pueden citar a la universidad de Cuenca, Técnica Particular de Loja y Escuela Politécnica del Litoral (ESPOL), todas cuentan con un centro de emprendimiento e innovación tecnológica; y utilizan técnicas modernas para aproximar a sus estudiantes a la vida emprendedora. Lo que está contribuyendo para que otros centros de educación superior comiencen a pensar en implementar estas iniciativas.

También menciona Feijoo que a pesar de todos los cambios estructurales que se ha realizado dentro de las universidades ecuatorianas para incentivar el emprendimiento, éstos no han sido suficientes para fortalecer el ímpetu de los estudiantes para emprender, dando como resultado estadísticas y resultados infértiles en muchos casos.

\section{Emprendimiento desde la Universidad Técnica de Machala}

La Universidad Técnica de Machala (UTMACH) desde el año 2014 viene apoyando el emprendimiento en la provincia de El Oro con la unidad de Vinculación, Cooperación, Pasantías y Prácticas (VINCOPP) sus datos no son tan claros, pero pueden demostrar la existencia de 31 proyectos aprobados para el 2014, con participación total de sus Unidades Académicas. El financiamiento de estos proyectos es explicado por dos fuentes: la primera consiste en el financiamiento interno; lo que la UTMACH destina para el proyecto en base a los pedidos recibidos desde sus unidades académicas, mientras que la segunda fuente radica en el financiamiento externo; constituido por el apoyo de entidades privadas.

El presupuesto total destinado al desarrollo de proyectos para el 2014 fue de $\$$ $748.716,05$ de los cuales la UTMACH financió el 71,11\% (\$532.448,83) mientras que terceros aportaron el 28,68\% (\$214.767,22), con lo que se puede evidenciar el fuerte apoyo a la investigación considerando que se encontraba en fase de categorización por parte del Consejo de Evaluación, Acreditación y Aseguramiento de la Calidad de la Educación Superior (CEAACES) y la demanda de recursos que se necesitaban para el efecto..

Para el año 2015, los proyectos aprobados bajan a 13, exponiendo una fuerte reducción respecto del año anterior, lo cual no tiene una explicación desde el organismo facilitador de la información como es VINCOPP. El presupuesto designado por la universidad para la ejecución de los mismos también disminuyó aunque no en gran medida (\$102.321,49 de diferencia). El presupuesto general total fue de \$ 589.343,02, donde la UTMACH aportó el 72,98\% (\$ $430.127,34)$ en tanto el apoyo de entidades privadas fue del 27,02\% $(\$ 159.215,68)$, en términos relativos a pesar de la disminución de proyectos el porcentaje de financiamiento crece con lo que se confirma el impulso a la investigación por parte de la universidad. 
2016 presenta de nuevo un descenso en el número de proyectos aprobados. Únicamente 11 fueron los aceptados, presupuestándose un total de $\$ 340.032,63$ para su realización, de estos el $47,77 \%$ (\$162.431,08) constituye el aporte de la universidad y 52,23\% (\$177.601,55) la contraparte. Sin embargo la contribución realizada por fuentes privadas de financiamiento para el año en cuestión es mayor que lo fijado por la UTMACH, ahora se puede observar una disminución porcentual importante comparada con los dos años anteriores del $72.98 \%$ se pasa al 47.77\% el aporte interno, sin una explicación sólida que permita sacar conclusiones sobre esta variación.

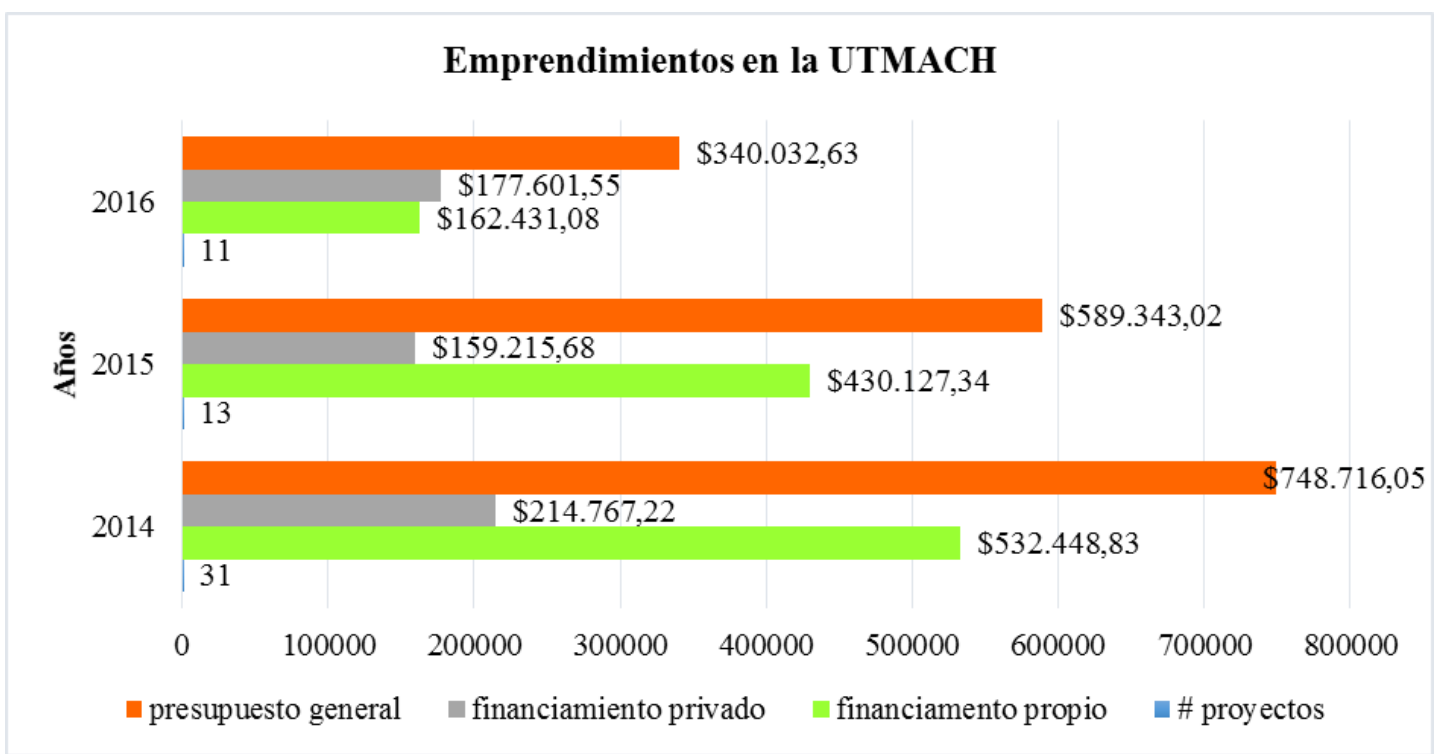

Figura 1. Evolución del presupuesto asignado por concepto de proyectos de emprendimiento. Datos proporcionados por VINCOPP-UTMACH.

En el año 2014 todas las unidades académicas de la universidad presentaron proyectos de modo individual y multidisciplinarios vinculantes entre estas unidades. Para los años 2015 y 2016, la situación cambia y sólo tres de las cinco unidades académicas se hacen presentes con proyectos individuales, incrementando incluso para el año 2016 el número de proyectos multidisciplinarios aceptados, lo que conlleva a pensar que hay una preferencia de las unidades académicas por trabajar colaborativamente que de forma particular, tratando de impactar de una manera potente en la investigación y en el emprendimiento derivado de la misma.

De los cincuentaicinco proyectos de vinculación con la comunidad, treintaiuno de ellos son considerados emprendimientos según la información proporcionada por VINCOPP, aunque no se tiene claridad en esta clasificación. Adicional a la inexistencia de información soporte que los considere como emprendimientos, tampoco se tienen desde VINCOPP el número de beneficiarios de los proyectos aprobados, por lo que es difícil determinar el impacto que han suscitado en su ejecución, o si lograron los objetivos planteados en su presentación.

De igual forma se investigó el apoyo al emprendimiento en la provincia por parte de la Universidad Metropolitana sede Machala institución con educación presencial, en la que no se pudo obtener cifras o datos estadísticas sobre emprendimiento o actividades relacionadas. Solo 
se indicó que cuentan con trabajos de pregrado que toman ciertas ideas de emprendimiento sin cuenten con proyectos de alto impacto creados en esta línea.

\section{Marco legal y apoyo del sector público para el emprendimiento}

Al revisar el marco legal del Ecuador se puede encontrar referencias muy importantes de apoyo al emprendimiento en la Ley Orgánica de Educación Superior (LOES), siendo los artículos 13, 24, 96 y 106 los que incentivan a dicha actividad, y que expresan como las universidades deben garantizar la vinculación con la sociedad, para que así puedan impulsar y promover la creación y desarrollo dentro del entorno social; así mismo declaran que es esencial asegurar la calidad educativa en vinculación con otras instituciones, que permitan crear sinergias y fusiones que promueva el desarrollo e innovación, los cuales vayan de acuerdo a expectativas y necesidades que presente la colectividad y planificación territorial. Además, la LOES establece que para la vinculación con los diferentes sectores de la sociedad se asigne a las universidades según ciertos criterios una parte de la distribución de recursos económicos por parte del Estado (Ley 298, 2010).

Con lo que VINCOPP de la UTMACH se convierte en la fuente apropiada para el análisis del emprendimiento. Dentro del marco regulatorio se pude hablar del proyecto de Ley Orgánica de Fomento al Emprendimiento, la que presenta incisos que favorecen e incentivan a la actividad emprendedora; debiendo mencionar que la misma se encuentra en observación por parte del pleno de la Asamblea Nacional del Ecuador desde el año 2013.

El sector público también ha cumplido un rol importante dentro del emprendimiento a nivel nacional, el cual a través de proyectos incentiva e impulsa las diferentes propuestas que presentan emprendedores. Desde lo público hay prestación de servicios y múltiples programas de apoyo para lograr gestar ideas; los ministerios de la Producción y de Inclusión Económica y Social, además instituciones como el Banco Nacional de Fomento (BNF) y la Corporación Nacional Financiera (CFN) son ejemplos de lo mencionado (Landsdale, Abad y Vera, 2012).

Estos ministerios e instituciones financieras, aportan con programas que apoyan al emprendimiento pudiendo mencionar: Ecuador Innova (programa de la Vicepresidencia de la República), Fondo de Innovación Productiva ${ }^{2}$ impulsado por el Ministerio de la Producción (MIPRO) destinado a invertir en diversos proyectos de innovación y emprendimiento en cuanto a servicios, productos y modernización tecnológica. Cabe mencionar que hasta hace pocos años atrás existían programas que aportaban al incentivo del emprendimiento, pero al no tener el éxito esperado fueron dados de baja, pudiendo mencionar al fondo El Cucayo de la Secretaría Nacional del Migrante (SENAMI), FONDEPYME, CreeEcuador e InnovaEcuador.

Dentro de la provincia de El Oro, en cuanto a lo gubernamental el Gobierno Provincial Autónomo de El Oro, por medio de la Corporación Orense de Desarrollo Económico y Territorial (CORPODET), ha incentivado y ayudado a varios proyectos de emprendimiento a través de un capital semilla desde el año 2012, actualmente de información proporcionada por esta institución pública existen dieciséis proyectos activos en diferentes sectores, pudiendo mencionarse: turismo, alimentos procesados, textiles, construcción y energías renovables.

\footnotetext{
${ }^{2}$ http://fondoinversion.industrias.gob.ec/
} 
Aunque también se adolece de información estadística de seguimiento temporal, por lo que no es posible determinar la supervivencia de los mismos y los problemas que puedan haber tenido.

\section{Conclusiones}

Al considerar las dos instituciones de educación superior en modalidad presencial que se asientan dentro de la provincia de El Oro, la Universidad Técnica de Machala evidencia a través del VINCOPP un registro de distintos proyectos que a decir de la información recibida fomentan el emprendimiento; sin embargo, este organismo no supo demostrar si existe algún seguimiento de los mismos para determinar cuál ha sido el impacto y beneficio que han generado dentro de la sociedad. Por otro lado, la Universidad Metropolitana, no cuenta con datos que los vinculen a la actividad emprendedora provincial, esta menciona la existencia de ciertos trabajos de titulación, pero sin embargo no existe el conocimiento si los mismos se han concretado como emprendimientos.

Existe un análisis por parte del GEM que hace referencia de cómo está el emprendimiento dentro del Ecuador, sin embargo este es muy general, ya que no existe un análisis o informe específico con respecto a la actividad emprendedora dentro de las universidades, por lo tanto, no se cuenta con una información concreta de organismos externos que hayan evaluado el impacto o la participación de las instituciones de educación superior para promover el emprendimiento.

Dentro de la provincia de El Oro, se realizó un recorrido en las instituciones públicas, de las cuales únicamente el Gobierno Provincial Autónomo de El Oro, evidencia escasos proyectos que incentiva a la actividad emprendedora, sin embargo, esta institución tampoco presenta un seguimiento adecuado de los mismos, en donde se pueda corroborar cual ha sido el nivel de incidencia dentro de la comunidad.

Desde el marco legal se encontró la existencia de organismos que a través de leyes impulsan la actividad emprendedora, como es el caso de la Ley Orgánica de Educación Superior, la cual dedica varios artículos que promueven dicha actividad, obligando o motivando a las universidades a que fomenten la vinculación de las diferentes Carreras con la sociedad. Así mismo se cuenta con una propuesta de ley que trata de dar mayores facilidades a las personas para emprender, estando en la actualidad calificada para prime debate.

\section{Bibliografía}

Bonaccorsi, A., \& Piccaluga, A. (1994). A theoretical framework for the evaluation of universityindustry relationships. $R \& D$ Management, 229-247.

Cardona, P., Morales, J., Cárdenas, M., \& Ramírez, L. (2015). Papel De La Educación Superior En Los Procesos De Formación En Emprendimiento. Sinapsis, 95. Obtenido de http://www.eam.edu.co/ojs-2.4.2/index.php/prueba/article/view/126

Castro, E., \& Vega, J. (Abril de 2009). Las relaciones universidad-entorno socioeconómico en el Espacio Iberoamericano del Conocimiento. Revista CTS, Vol. 4(n $\left.{ }^{\circ} 12\right), 72-74$. Obtenido de http://www.scielo.org.ar/scielo.php?pid=S185000132009000100008\&script=sci_arttext 
Chaparro, F. (2010). Universidad, creación de conocimiento, innovación y desarrollo. Ciencia, tecnología y universidad en Iberoamérica, 45-46. Obtenido de http://blogs.ua.pt/isabelpmartins/bibliografia/ciencia_tecnologia\%20_universidad_iberoa merica.pdf\#page $=45$

Ecuador, A. N. (12 de Octubre de 2010). Ley Orgánica de Educación Superior. Registro Oficial 298. Quito, Pichincha, Ecuador: Registro Oficial Organo del Gobierno del Ecuador. Obtenido de http://www.asambleanacional.gob.ec/es/system/files/ley_organica_educacion_superior.pd $\mathrm{f}$

Feijoo, N. (2015). Diseño de una red de vinculación entre empresarios, universidades y organizaciones del Estado para el fortalecimiento del desarrollo emprendedor de la provincia de Manabí. Universidad Privada Antenor Orrego, 32-33. Obtenido de http://repositorio.upao.edu.pe/bitstream/upaorep/731/1/Feijo_Nilba_Red_Vinculaci\%C3 $\% 93 n \_$Empresarios.pdf

Fernández de Lucio, I., Castro, E., Conesa, F., \& Gutiérrez, A. (2000). Las relaciones universidadempresa: entre la transferencia de resultados y el aprendizaje regional. Espacios. Obtenido de http://digital.csic.es/bitstream/10261/13382/1/Relaciones_universidad_empresa.pdf

García, A., Cantón, L., \& Torreblanca, Á. (2015). Evaluación por competencias de un programa educativo emprendedor universitario. Revista Iberoamericana de Contaduría, Economía y Administración, $2 . \quad$ Obtenido de https://dialnet.unirioja.es/servlet/articulo?codigo $=5263331$

GEM. (2015). Comparación del entorno emprendedor entre Ecuador, países de América Latina y economías de eficiencia. Reino Unido: GEM.

Huanca, R. (2004). La investigación universitaria de países en desarrollo y la visión de los académicos sobre la relación universidad empresa: universidades públicas de la región occidental de Bolivia. Valencia: Universidad Politécnica de Valencia: Departamento de Organización de Empresas, Economía Financiera y Contabilidad. Obtenido de https://riunet.upv.es/bitstream/handle/10251/2672/tesisUPV2149.pdf?sequence=1\&isAllo wed $=\mathrm{y}$

Juliá, J. (2014). Tribuna de opinión: emprendimiento y universidad. Una referencia al caso de España y a la UPV. REVESCO, 18-21. Obtenido de https://dialnet.unirioja.es/servlet/articulo?codigo $=5000922$

Krauss, C. (2011). Actitudes emprendedoras de los estudiantes universitarios: El caso de la Universidad Católica del Uruguay. Dimensión empresarial, 28-40.

Landsdale, D., Abad, C., \& Vera, D. (2012). Impulsores claves para establecer el ecosistema dinámico de emprendimiento en Ecuador. Polémika, 21. Obtenido de 
http://www.usfq.edu.ec/publicaciones/polemika/Documents/polemika009/polemika009_0 07_articulo003.pdf

Ortín, P., Salas, V., Trujillo, M., \& Vendrell, F. (2008). La creación de spin-off universitarias en España: características, determinantes y resultados. Economía Industrial, 79-81. Obtenido de http://dialnet.unirioja.es/servlet/articulo? codigo=2671578\&info=resumen\&idioma=ENG

Pertuz, V., Rojas, G., Navarro, A., \& Quintero, L. (2016). Perfi 1 docente y fomento de la cultura del emprendimiento: búsqueda de una relación. Educación y educadores, 33. Obtenido de https://dialnet.unirioja.es/servlet/articulo?codigo $=5558830$

Piguave, V. (2014). Importancia del desarrollo de la creatividad para los estudiantes de la carrera de Ingeniería Comercial desde el proceso de enseñanza-aprendizaje. Educación, 32. Obtenido de https://dialnet.unirioja.es/servlet/articulo?codigo=5056841

Poblete, C., \& Amorós, J. (2013). University Support in the Development of Regional Entrepreneurial Activity: An Exploratory Study from Chile. Investigaciones Regionales, 162. Obtenido de https://dialnet.unirioja.es/servlet/articulo?codigo=4459812

Rodríguez, M., \& Lasio, V. (2007). Educación Formal y Empresa Juvenil: Contraste de Dos Enfoques para Desarrollar el Espíritu Emprendedor. Latin American and Caribbean Journal of Engineering Education, 89. Obtenido de http://laccei.eng.fau.edu/index.php/lacjee/article/view/17/15

Sanabria, P., Morales, M., \& Ortiz, C. (2015). Interacción universidad y entorno: marco para el emprendimiento. Educación y Educadores, 114-116. Obtenido de https://dialnet.unirioja.es/servlet/articulo?codigo=5429699

Thursby, M. C. (2005). Introducing Technology Entrepreneurship to Graduate Education: An Integrative Approach. Advances in the Study of Entrepreneurship, Innovation, and Economic Growth, 211-240.

Yachay Tech. (22 de 04 de 2014). www.yachaytech.edu.ec. Obtenido de http://www.yachaytech.edu.ec/fileadmin/user_upload/uploads/LOTAIP/Estatuto_de_la_ Universidad_de_Investigacion_de_Tecnologia_Experimental_YACHAY.pdf

\section{Apéndice}

Tabla 1

Proyectos de vinculación con la sociedad aprobados 2014

Unidades académicas
Número de proyectos
Financiamiento

propio
Financiamiento

privado
Presupuesto

general

Año 2014 


\begin{tabular}{|c|c|c|c|c|}
\hline UACA & 7 & $\$ 78.285,00$ & $\$ 46.160,00$ & $\$ 124.445,00$ \\
\hline UACE & 5 & $\$ 117.373,95$ & $\$ 119.446,00$ & $\$ 236.819,95$ \\
\hline UACQS & 5 & $\$ 181.984,00$ & $\$ 21.310,00$ & $\$ 203.294,00$ \\
\hline UACS & 7 & $\$ 82.607,20$ & $\$ 11.620,00$ & $\$ 94.227,20$ \\
\hline UAIC & 1 & $\$ 9.810,00$ & $\$ 6.016,22$ & $\$ 15.826,22$ \\
\hline Proyectos multidisciplinarios & 4 & $\$ 34.884,50$ & $\$ 8.085,00$ & $\$ 42.969,50$ \\
\hline Sin especificar & 2 & $\$ 27.504,18$ & $\$ 3.630,00$ & $\$ 31.134,18$ \\
\hline Total general & 31 & $\$ 532.448,83$ & $\$ 214.767,22$ & $\$ 748.716,05$ \\
\hline
\end{tabular}

Tabla 2

Proyectos de vinculación con la sociedad aprobados 2015

\begin{tabular}{lcrrr}
\hline \multicolumn{1}{c}{ Unidades académicas } & Número de proyectos & $\begin{array}{c}\text { Financiamiento } \\
\text { propio }\end{array}$ & $\begin{array}{c}\text { Financiamiento } \\
\text { privado }\end{array}$ & $\begin{array}{c}\text { Presupuesto } \\
\text { general }\end{array}$ \\
\hline Año 2015 & 4 & $\$ 69.147,75$ & $\$ 83.290,48$ & $\$ 152.438,23$ \\
UACE & 1 & $\$ 5.150,00$ & $\$ 840,00$ & $\$ 5.990,00$ \\
UACQS & 4 & $\$ 199.898,31$ & $\$ 41.210,00$ & $\$ 241.108,31$ \\
UACS & 4 & $\$ 155.931,28$ & $\$ 33.875,20$ & $\$ 189.806,48$ \\
Proyectos multidisciplinarios & 13 & $\$ 430.127,34$ & $\$ 159.215,68$ & $\$ 589.343,02$ \\
Total general & & & & \\
\hline
\end{tabular}

Nota: UACE = Unidad Académica de Ciencias Empresariales; UACQS = Unidad Académica de Ciencias Químicas y de la Salud; UACS = Unidad Académica de Ciencias Sociales.

Tabla 3

Proyectos de vinculación con la sociedad aprobados 2016

\begin{tabular}{lcrrr}
\hline \multicolumn{1}{c}{ Unidades académicas } & Número de proyectos & $\begin{array}{c}\text { Financiamiento } \\
\text { propio }\end{array}$ & $\begin{array}{c}\text { Financiamiento } \\
\text { privado }\end{array}$ & $\begin{array}{c}\text { Presupuesto } \\
\text { general }\end{array}$ \\
\hline Año 2016 & & & & \\
UACA & 1 & $\$ 20.056,80$ & $\$ 960,00$ & $\$ 21.016,80$ \\
UACE & 2 & $\$ 37.922,30$ & $\$ 1.464,00$ & $\$ 39.386,30$ \\
UACQS & 1 & $\$ 7.493,35$ & $\$ 20.198,00$ & $\$ 27.691,35$ \\
\hline
\end{tabular}




\begin{tabular}{lcrrr}
\hline Proyectos multidisciplinarios & 7 & $\$ 96.958,63$ & $\$ 154.979,55$ & $\$ 251.938,18$ \\
Total general & 11 & $\$ 162.431,08$ & $\$ 177.601,55$ & $\$$ \\
& & & & $340.032,63$ \\
\hline
\end{tabular}

Nota: UACA = Unidad Académica de Ciencias Agropecuarias; UACE = Unidad Académica de Ciencias Empresariales; UACQS = Unidad Académica de Ciencias Químicas y de la Salud. 